Geom. Funct. Anal. Vol. 20 (2010) 1306

DOI 10.1007/s00039-010-0100-9

Published online November 6, 2010

(C) 2010 Springer Basel AG

GAFA Geometric And Functional Analysis

\title{
ERRATUM TO: BILLIARDS IN L-SHAPED TABLES WITH BARRIERS
}

\author{
Matt BAINBRIDGe
}

Erratum to: Geom. Funct. Anal. Vol. 20 Nr. 2 (2010), 299-356,

DOI 10.1007/s00039-010-0065-8

In this erratum, we point out a gap in the proof of Theorem 1.1 of [B]. In Theorem 12.2, we stated a classification of nondivergent, ergodic, $h_{t}$-invariant measures on the eigenform locus $\mathcal{E}_{D}$. By nondivergent, we implicitly meant nondivergent under the positive-time geodesic flow $a_{t}$. This is a mistake; the classification stated is actually of measures which are nondivergent under the negative-time geodesic flow. In the first paragraph of Lemma 12.3, we then assume that we are working with the positive-time geodesic flow in the statement "any subsequential limit of $m_{x}^{t}$ is $h_{t}$-invariant."

This incorrect argument was intended to get around the fact that the classification of ergodic $h_{t}$-invariant measures on the eigenform locus from [CW] was incomplete. Theorem 1.1 should remain true, but a correct proof seems to require a complete classification of ergodic $h_{t}$-invariant measures. For now, Theorem 1.1 should be regarded as a conjecture. It does follow from [EM] that the asymptotics of Theorem 1.1 hold for almost every surface in the eigenform locus $\Omega E_{D}(1,1)$.

I would like to thank Barak Weiss for pointing out this mistake.

\section{References}

[B] M. Bainbridge, Billiards in l-shaped tables with barriers, Geom. Funct. Anal. $20: 2$ (2010), 299-356.

[CW] K. Calta, K. Wortman, On unipotent flows in $\mathcal{H}(1,1)$ Ergodic Theory Dynam. Systems 30:2 (2010), 379-398.

[EM] A. Eskin, H. Masur, Asymptotic formulas on flat surfaces, Ergodic Theory Dynam. Systems 21:2 (2001), 443-478.

Matt Bainbridge, Department of Mathematics, Indiana University, Rawles Hall, 831 East 3rd St, Bloomington, IN 47405, USA

The online version of the original article can be found under doi:10.1007/s00039-010-0065-8. 